DFPD/2017/TH/02

\title{
Supersymmetric Black Holes and Freudenthal Duality
}

\author{
Taniya Mandal ${ }^{1,2}$, Alessio Marrani ${ }^{3,4}$ and Prasanta K. Tripathy ${ }^{1}$ \\ ${ }^{1}$ Department of Physics, Indian Institute of Technology Madras, \\ Chennai 600036, India \\ ${ }^{2}$ Institute of Mathematical Sciences, C.I.T Campus, Taramani \\ Chennai 600113, India \\ ${ }^{3}$ Museo Storico della Fisica e Centro Studi e Ricerche "Enrico Fermi", \\ Via Panisperna 89A, I-00184, Roma, Italy \\ ${ }^{4}$ Dipartimento di Fisica e Astronomia "Galileo Galilei", Università di Padova, \\ and INFN, Sez. di Padova, \\ Via Marzolo 8, I-35131 Padova, Italy \\ taniya@physics.iitm.ac.in, alessio.marrani@pd.infn.it, prasanta@iitm.ac.in
}

\begin{abstract}
We study the effect of Freudenthal duality on supersymmetric extremal black hole attractors in $\mathcal{N}=2, D=4$ ungauged supergravity. Freudenthal duality acts on the dyonic black hole charges as an anti-involution which keeps the black hole entropy and the critical points of the effective black hole potential invariant. We analyze its effect on the recently discovered distinct, mutually exclusive phases of axionic supersymmetric black holes, related to the existence of non-trivial involutory constant matrices. In particular, we consider a supersymmetric $D 0-D 4-D 6$ black hole and we explicitly Freudenthal-map it to a supersymmetric $D 0-D 2-D 4-D 6$ black hole. We thus show that the charge representation space of a supersymmetric $D 0-D 2-D 4-D 6$ black hole also contains mutually exclusive domains.
\end{abstract}




\section{Introduction}

Black holes always remain a topic of great interest because of their thermodynamic behavior. It is one of the significant success of string theory to provide statistical description (by counting degeneracy of $D$-branes in the weak coupling limit) of the macroscopic (Bekenstein-Hawking) entropy [1] of certain supersymmetric black holes.

A crucial phenomenon characterizing certain classes of black holes is the attractor mechanism, which states that, for an extremal black hole, scalar fields are drawn towards fixed values at the horizon losing all asymptotic memories; such fixed values depend only on black hole electric and magnetic charges [2]. A static, spherically symmetric and asymptotically flat black hole can be described by an effective one-dimensional theory. The attractors are then obtained by extremizing the effective black hole potential, that is an algebraic function of the moduli fields and of the charges. The horizon value of the effective potential provides the black hole entropy, which depends only on the black hole charges [3].

An interesting issue in black hole physics concerns the multiplicity of the attractors within the moduli space; this was first investigated by Moore [4]. In $\mathcal{N}=2, D=5$ ungauged supergravity, existence of multiple supersymmetric ( $\frac{1}{2}$-BPS) attractors has been discussed and explicitly constructed for a two-moduli, non-homogeneous model [5]. In this case, multiplicity arises due to the presence of disjoint branches in the moduli space [6] (thus being consistent with the uniqueness results of [7]). Using the correspondence between $4 D / 5 D$ critical points [8], the analysis has been reduced to four dimensions and it has been shown that a five-dimensional multiple supersymmetric attractor leads to one supersymmetric and one non-supersymmetric critical point in four dimensions [9]. While in $D=4$ the supersymmetric attractor is proved to be unique [10], there exist multiple non-supersymmetric attractors with the same charge configurations, the same entropy and the same number of zero modes to the mass matrix (massless Hessian modes); it is puzzling to note that such multiple solutions exist also when the moduli space is connected [9]. By introducing particular involutory constant matrices (generally depending on the geometry of the moduli space), in [10] it has been also shown that the representation space of e.m. charges of four dimensional supersymmetric black holes with non-vanishing axions contains mutually exclusive domains, and inside each domain the attractor is unique. Within the same framework, new phases of non-BPS attractors were recently discovered in [11].

As the black hole charges transform linearly under symplectic transformations of the (electric-magnetic) $U$-duality group 1 , the entropy is a $U$-duality invariant quantity. Four dimensional extremal black holes also exhibit another transformation which keeps the entropy invariant, called Freudenthal duality (F-duality). F-duality can be defined as an anti-involutive, non-linear map acting on symplectic spaces, in particular on the representation space of the black hole electric-magnetic charges (which becomes a charge lattice upon quantization). After its introduction [14], in [15] F-duality was shown to be a sym-

\footnotetext{
${ }^{1}$ Throughout the present investigation, we work in the (semi)classical regime for which the electromagnetic charges take values in the real numbers. Here $U$-duality is referred to as the "continuous" symmetries of [12. Their discrete versions are the non-perturbative $U$-duality string theory symmetries studied in [13].
} 
metry not only of the classical Bekenstein-Hawking entropy, but also of the critical points (attractors) of the effective black hole potential itself (see also [16, 17]). Moreover, it was extended to any generalized special geometry, thus comprising all $\mathcal{N}>2$ (extended) supergravities, as well as $\mathcal{N}=2$ generic, not necessarily homogeneous special Kähler geometry [15]. It is here worth remarking that F-duality appeared in various other contexts, such as gauge theories with symplectic scalar manifolds [18] and multi-centered black holes [19]. Lagrangian densities with on-shell F-duality symmetry were constructed in [20]. Finally, in [21] F-duality was consistently formulated in the context of Abelian gaugings of $\mathcal{N}=2$, $D=4$ supergravity, both for $U(1)$ Fayet-Iliopoulos gauging and for theories coupled to hypermultiplets.

Despite preserving homogeneity in charges, F-duality is an intrinsically non-linear, antiinvolutive map acting on charges, thus it is inherently different from $U$-duality, and issues of higher derivative corrections are not yet well understood. By denoting with $n+1$ the number of Abelian vector fields, F-duality acts on the dyonic charge vector $Q^{M}=\left(p^{\Lambda}, q_{\Lambda}\right)$ $(\Lambda=0,1, \ldots, n)$ as an entropy-preserving anti-involution [15]

$$
\begin{aligned}
\pi \hat{Q}^{M}(Q) & :=\Omega^{M N} \frac{\partial S(Q)}{\partial Q^{N}} \\
\widehat{\hat{Q}} & =-Q \\
S(Q) & =S(\hat{Q})
\end{aligned}
$$

where $\Omega$ is the $2(n+1) \times 2(n+1)$ symplectic metric: $\Omega^{M N}=\left(\begin{array}{cc}0 & -I \\ I & 0\end{array}\right), \Omega^{T}=-\Omega$ and $\Omega^{2}=-\mathbb{I}$, and $S$ is the Bekenstein-Hawking (semi)classical black hole entropy. Fduality generally commutes with supersymmetry (if any) and with $U$-duality; note that no assumption on the geometry of the moduli space of the scalar fields has been made.

Within the context of cubic geometries of $\mathcal{N}=2, D=4$ ungauged supergravity coupled to $n$ vector multiplets (usually arising from the large volume limit of compactifications of Type IIA superstrings on Calabi-Yau manifolds), the aim of this paper is to study the effect of F-duality on the the most general, axionic $D 0-D 4-D 6$ supersymmetric black hole solution, which was obtained in [10] by introducing suitable constant, involutory matrices. Considering a simple (yet, non-homogeneous) $n=2$ model, it was shown that the symplectic representation space of dyonic black hole charges (which becomes a charge lattice upon imposing Dirac-Schwinger-Zwanzinger quantization conditions) contains mutually exclusive domains, and that inside each domain the attractor solution is unique [10]. By F-transforming the most general $D 0-D 4-D 6$ BPS black hole attractors, we will prove that such mutually exclusive domains also exist for the most general $D 0-D 2-D 4-D 6$ BPS supporting charge configuration. Moreover, we will formulate the most general Ansatz for the entropy and the value of the horizon, attracted moduli within such a configuration, thus fully generalizing the results of [22] and [10].

The plan of the paper is as follows. 
In Sec. 2.1, we recap the $D 0-D 4-D 6$ BPS black hole attractor solution, while in Sec. 2.2 we consider a model with two moduli and state the condition of existence of a non-trivial involutory matrix. We further comment on the non-homogeneity and explicit realization of such a model, as well as on the $U$-invariance, in Sec. 2.2.1.

Then, in Sec. 3 we apply F-duality on the $D 0-D 4-D 6$ BPS black hole, finding that the corresponding F-dual configuration is a $D 0-D 2-D 4-D 6$ BPS black hole.

Sec. 4 is devoted to the generalization of the results of [22] and [10] : we discuss the most general supersymmetric $D 0-D 2-D 4-D 6$ black hole attractor solution which, for particular values of real, charge-dependent parameters, is F-dual to $D 0-D 4-D 6$.

Sec. 5 presents some comments and hints for further developments.

An Appendix, containing some technical details, concludes the paper.

\section{Supersymmetric $D 0-D 4-D 6$ Black Hole}

\subsection{The Solutions}

Attractor solutions describing supersymmetric extremal $D 0-D 4-D 6$ black holes in $\mathcal{N}=2, D=4$ supergravity have been studied in [10]. In a special Kähler geometry of the vector multiplets' moduli space described by the holomorphic prepotential $(a=1, \ldots, n)$

$$
F(X)=D_{a b c} \frac{X^{a} X^{b} X^{c}}{X^{0}}
$$

the most general supersymmetric attractor solution to this black hole configuration is given by

$$
\begin{aligned}
& x_{1}^{a}=\frac{1}{p^{0}}\left(p^{a}-\frac{2 D-q_{0}\left(p^{0}\right)^{2}}{2 \chi} I_{b}^{a} p^{b}\right) \\
& x_{2}^{a}=-\frac{1}{p^{0}} \sqrt{1-\left(\frac{2 D-q_{0}\left(p^{0}\right)^{2}}{2 \chi}\right)^{2}} I_{b}^{a} p^{b},
\end{aligned}
$$

where the so-called $4 D / 5 D$ special coordinates' symplectic frame is used2, with the scalar fields denoted by $x^{a}:=x_{1}^{a}+i x_{2}^{a}$. Moreover, we used the notation of [10], namely $D:=$ $D_{a b c} p^{a} p^{b} p^{c}, D_{a}:=D_{a b c} p^{b} p^{c}$ and $\chi:=D_{a} I_{b}^{a} p^{b}$. The involutory matrix $I_{b}^{a}$ satisfies [10]

$$
\begin{aligned}
I_{c}^{a} I_{b}^{c} & =\delta_{b}^{a} \\
D_{a d e} I_{b}^{d} I_{c}^{e} & =D_{a b c} .
\end{aligned}
$$

The corresponding black hole entropy takes the following form [10]:

$$
S=\frac{\pi}{p^{0}} \sqrt{4 \chi^{2}-\left(2 D-q_{0} p^{0^{2}}\right)^{2}} .
$$

\footnotetext{
${ }^{2}$ In such a symplectic frame, the $D$-brane charges can be denoted as follows : $D 0\left(q_{0}\right), D 2\left(q_{a}\right), D 4$ $\left(p^{a}\right)$ and $D 6\left(p^{0}\right)$, as understood throughout.
} 
Any choice of $I^{a}{ }_{b}$ that satisfy conditions (2.4)-(2.5) will provide an independent and welldefined expression of the Bekenstein-Hawking entropy and of corresponding attractor, horizon values of the scalar fields (2.2)-(2.3). The trivial choice of the involution is of course $I^{a}{ }_{b}=\delta^{a}{ }_{b}$. For this choice, (2.6) reduces to

$$
S=\pi \sqrt{4 q_{0} D-\left(q_{0} p^{0}\right)^{2}},
$$

which is the standard entropy of $D 0-D 4-D 6$ BPS extremal black hole [22]. The attractor value of the scalar fields for this trivial choice of involutory matrix is 3 [10, 22,

$$
x^{a}=\frac{p^{a}}{2 D}\left(p^{0} q_{0}-i \sqrt{4 q_{0} D-\left(p^{0} q_{0}\right)^{2}}\right) .
$$

Note that the above expression reduces to the smooth $D 0-D 4$ attractor solution upon setting the $D 6$ charge $p^{0}$ to zero. However, for any other choice of $I^{a}{ }_{b}$ the solutions (2.2) and (2.3) as well as the corresponding entropy (2.6) become singular in the limit $p^{0} \rightarrow 0$. Thus, these new branches of solutions cease to exist in the absence of $D 6$ branes. This is consistent with the uniqueness of $D 0-D 4$ attractors [10].

\section{2 $n=2$ Model and Non-trivial Involutory Matrix}

By considering an example of two-moduli $(n=2)$ model, in Sec. 4 of [10], it has also been shown explicitly that there exists a non-trivial choice of an involutory matrix $I^{a}{ }_{b} \neq \delta^{a}{ }_{b}$ satisfying (2.4)-(2.5) . A generic $2 \times 2$ involutory matrix can be parametrized as

$$
I_{b}^{a}=:\left(\begin{array}{cc}
u & v \\
w & -u
\end{array}\right),
$$

such that $u^{2}+v w=1$; for brevity's sake, we further denote the four possible components of the rank-3 completely symmetric tensor $D_{a b c}$ occurring in (2.1) (namely, of the triple intersection number of the Calabi-Yau manifold in the large volume limit of the Type IIA compactifications) as $D_{111}=a, D_{112}=b, D_{122}=c$ and $D_{222}=d$. It has been proved that the conditions (2.4) -(2.5) can be satisfied with $I^{a}{ }_{b} \neq \delta^{a}{ }_{b}$, for

$$
u=\frac{\mathcal{L}}{\sqrt{\mathcal{L}^{2}-4 \mathcal{M N}}}, v=\frac{-2 \mathcal{M}}{\sqrt{\mathcal{L}^{2}-4 \mathcal{M N}}}, w=\frac{2 \mathcal{N}}{\sqrt{\mathcal{L}^{2}-4 \mathcal{M N}}},
$$

where we introduced the notation $\mathcal{L}=a d-b c, \mathcal{M}=c^{2}-b d$ and $\mathcal{N}=b^{2}-a c$. Note that a crucial consistency condition is

$$
\mathcal{L}^{2}-4 \mathcal{M N}>0 .
$$

\footnotetext{
${ }^{3}$ From the treatment of $\left[8, \operatorname{Im}\left(x^{a}\right)<0\right.$ in the $4 D / 5 D$ special coordinates' symplectic frame of special Kähler geometry.
} 
For the choice (2.9) (2.10) of $I^{a}{ }_{b}$, the horizon, attractor values of the two vector multiplet moduli $x^{1}=x_{1}^{1}+i x_{2}^{1}$ and $x^{2}=x_{1}^{2}+i x_{2}^{2}$ are given by (2.2)-(2.3), which explicitly read

$$
\begin{aligned}
& x_{1}^{1}=\frac{1}{p^{0}}\left[p^{1}-\frac{\left(D-\frac{1}{2} q_{0}\left(p^{0}\right)^{2}\right)\left(\mathcal{L} p^{1}-2 \mathcal{M} p^{2}\right)}{\chi \sqrt{\mathcal{L}^{2}-4 \mathcal{M N}}}\right], \\
& x_{2}^{1}=-\frac{1}{p^{0}} \sqrt{1-\left(\frac{D-\frac{1}{2} q_{0}\left(p^{0}\right)^{2}}{\chi}\right)^{2}} \frac{\left(\mathcal{L} p^{1}-2 \mathcal{M} p^{2}\right)}{\sqrt{\mathcal{L}^{2}-4 \mathcal{M N}}}, \\
& x_{1}^{2}=\frac{1}{p^{0}}\left[p^{2}-\frac{\left(D-\frac{1}{2} q_{0}\left(p^{0}\right)^{2}\right)\left(2 \mathcal{N} p^{1}-\mathcal{L} p^{2}\right)}{\chi \sqrt{\mathcal{L}^{2}-4 \mathcal{M N}}}\right] \\
& x_{2}^{2}=-\frac{1}{p^{0}} \sqrt{1-\left(\frac{D-\frac{1}{2} q_{0}\left(p^{0}\right)^{2}}{\chi}\right)^{2}} \frac{\left(2 \mathcal{N} p^{1}-\mathcal{L} p^{2}\right)}{\sqrt{\mathcal{L}^{2}-4 \mathcal{M N}}} .
\end{aligned}
$$

So, for an $n=2$ model satisfying (2.11), two distinct and independent BPS attractor solutions exist, depending on whether $I^{a}{ }_{b}=\delta_{b}^{a}$ or $I_{b}^{a}$ given by (2.9) (2.10) is chosen.

Now the key question naturally arises whether these two BPS solutions exist for the same supporting $D 0-D 4-D 6$ black hole charge configuration. As investigated in [10], the conditions of positive definiteness of the Kähler metric $g_{a \bar{b}}$ for the two classes of BPS solutions boil down to the study of the sign of the quantity

$$
\mathcal{N}\left(p^{1}\right)^{2}-\mathcal{L} p^{1} p^{2}+\mathcal{M}\left(p^{2}\right)^{2}
$$

and they read as follows :

$$
\begin{aligned}
\text { 'std.' sol. (2.7)-(2.8): } & \mathcal{N}\left(p^{1}\right)^{2}-\mathcal{L} p^{1} p^{2}+\mathcal{M}\left(p^{2}\right)^{2}>0 \\
\text { 'non-std.' sol. (2.6) and (2.12) }-(2.15): & \mathcal{N}\left(p^{1}\right)^{2}-\mathcal{L} p^{1} p^{2}+\mathcal{M}\left(p^{2}\right)^{2}<0
\end{aligned}
$$

Also the positive definiteness of the gauge kinetic terms can (at least numerically) be checked to hold for both solutions for such distinct conditions. Thus, in the considered $n=2$ model, the 'standard' and 'non-standard' BPS attractor solutions are supported by two different, distinct domains in the representation space of the dyonic black hole charges [10].

\subsubsection{Non-Homogeneity and $U$-Invariance}

Some comments are in order here.

1. The consistency condition (2.11) necessarily implies the non-homogeneity of the vector multiplets's two-dimensional special Kähler moduli space. Indeed, from the classification of homogeneous $(d$-)spaces [23,24], the unique $n=2$ homogeneous model is 
the so-called $S T^{2}$ model (corresponding to the dimensional reduction of $(1,0)$, chiral minimal "pure" supergravity from $D=6$ to $D=4$ [24]), characterized by $c=2$ and $a=b=d=0$, and thus violating (2.11), because in this case $\mathcal{L}^{2}-4 \mathcal{M N}=0$. Thus, the $n=2$ model considered here, as well as in [10], has a non-homogeneous moduli space.

2. For explicit $n=2$ models for which the treatment of Sec. 2.2 holds, one can e.g. see the two Tables at pages 51-53 of [25], providing various (non-homogeneous) cubic models in which multiple axionic $D=4$ BPS attractors exist. For instance, $X_{14}^{I I}(7,2,2,2,1)$, for which $a=2, b=7, c=21, d=63$ is an example of such models [25].

3. As also resulting from the treatment e.g. of [24, all special Kähler geometries described by the cubic holomorphic prepotential (2.1) (usually named $d$-geometries) are characterized by a 'minimal' electric-magnetic $(U$-)duality symmetry, which pertains to (the large volume limit of) Calabi-Yau compactifications ( [26]; for recent accounts, cfr. [27] and [28], and Refs. therein). It can easily be checked that the two inequality conditions appearing in the r.h.s.'s of (2.17) and (2.18) are not invariant under such a duality, and thus they are not well-defined. In fact, in order to $U$-invariantly characterize the separation of the two domains in the charge representation space, one should better consider the positivity condition of the determinant of the metric $g_{a \bar{b}}$ for both classes of BPS solutions, respectively given by Eqs. (4.8) and (4.9) of [10], which are consistently $U$-invariant.

\section{Freudenthal Dual of $D 0-D 4-D 6$ BPS Black Hole}

In this section we apply F-duality on the $D 0-D 4-D 6$ charge vector $Q$ and find a new dyonic charge vector $\hat{Q}$. Following (1.1) and also the relation between moduli and entropy (cfr. e.g. [29, and Refs. therein)

$$
x^{a}=\frac{p^{a}+\frac{i}{\pi} \frac{\partial S}{\partial q_{a}}}{p^{0}+\frac{i}{\pi} \frac{\partial S}{\partial q_{0}}},
$$

we obtain the new F-transformed set of charges $\hat{Q}=\left(\hat{p}^{0}, \hat{p}^{a}, \hat{q}_{0}, \hat{q}_{a}\right)$ in terms of $Q=$ $\left(p^{0}, p^{a}, q_{0}, 0_{a}\right)$, where $S$ is the most general supersymmetric entropy [22] :

$$
\begin{aligned}
\hat{p}^{0} & =\frac{\pi\left(q_{0} p^{0^{2}}-2 D\right)}{S}, \\
\hat{p}^{a} & =\frac{\pi}{p^{0} S}\left[2 \chi I^{a}{ }_{b} p^{b}-\left(2 D-q_{0}\left(p^{0}\right)^{2}\right) p^{a}\right], \\
\hat{q}_{0} & =-\frac{\pi}{p^{0} S}\left[4 \chi^{2}-4 D^{2}+q_{0}^{2}\left(p^{0}\right)^{4}\right], \\
\hat{q}_{a} & =\frac{6 \pi}{p^{0^{2}} S}\left[2 \chi D_{b} I_{a}^{b}-\left(2 D-q_{0}\left(p^{0}\right)^{2}\right) D_{a}\right] .
\end{aligned}
$$


So, we have obtained a generic charge configuration (with all types of $D$-brane charges switched on) by acting with F-duality on the supersymmetric $D 0-D 4-D 6$ black hole : this is provided by the most general F-dualized charge configuration $\hat{Q}$ coming from supersymmetric $D 0-D 4-D 6$ black hole charge configuration $Q$.

\section{Supersymmetric $D 0-D 2-D 4-D 6$ Black Hole}

By further generalizing the results of [10], in this section we will find out the most general expression of entropy and moduli of a supersymmetric $D 0-D 2-D 4-D 6$ black hole, and show that it is F-dual to the supersymmetric $D 0-D 4-D 6$ with the help of Eqs. (3.2)(3.5). For convenience's sake, we will denote all generic BPS dyonic D0 - D2-D4-D6 charges, as well as the symplectic vector comprising them all, as tilded.

In order to find out the entropy and attractor solution, we follow the method due to Shmakova 22. For a generic supersymmetric black hole in Type IIA string theory with all non-vanishing $D$-brane charges, the entropy is given by [22] :

$$
\frac{S(\tilde{Q})}{\pi}=\frac{1}{3 \tilde{p}^{0}} \sqrt{\frac{4}{3}\left(\tilde{\Delta}_{a} \tilde{x}^{a}\right)^{2}-9\left(\tilde{p}^{0}(\tilde{p} \cdot \tilde{q})-2 \tilde{D}\right)^{2}},
$$

where $\tilde{\Delta}_{a}:=3 \tilde{D}_{a}-\tilde{p}^{0} \tilde{q}_{a}, \tilde{D}_{a}:=D_{a b c} \tilde{p}^{b} \tilde{p}^{c}, \tilde{D}:=\tilde{D}_{a} \tilde{p}^{a}$ and $\tilde{p} \cdot \tilde{q}:=\tilde{p}^{0} \tilde{q}_{0}+\tilde{p}^{a} \tilde{q}_{a}$. The variables $\tilde{x}^{a}$ are the real solutions of

$$
D_{a b c} \tilde{x}^{b} \tilde{x}^{c}=\tilde{\Delta}_{a} .
$$

The moduli fields $x^{a}=x_{1}^{a}+i x_{2}^{a}$ at the BPS attractor point are [22]

$$
\begin{aligned}
x_{1}^{a} & =\frac{3}{2} \frac{\tilde{x}^{a}}{\tilde{p}^{0}\left(\tilde{\Delta}_{c} \tilde{x}^{c}\right)}\left(\tilde{p}^{0}(\tilde{p} \cdot \tilde{q})-2 \tilde{D}\right)+\frac{\tilde{p}^{a}}{\tilde{p}^{0}}, \\
x_{2}^{a} & =-\frac{3}{2} \frac{\tilde{x}^{a}}{\left(\tilde{\Delta}_{c} \tilde{x}^{c}\right)} \frac{S(\tilde{Q})}{\pi} .
\end{aligned}
$$

To solve Eq. (4.2), we consider the most general Ansatz

$$
\tilde{x}^{a}=\alpha \tilde{p}^{0} I^{a}{ }_{b} \tilde{D}^{b c} \tilde{q}_{c}+\beta \tilde{p}^{0} \tilde{D}^{a b} \tilde{q}_{b}+\sigma I^{a}{ }_{b} \tilde{p}^{b}+\rho \tilde{p}^{a},
$$

where $\tilde{D}^{a c} \tilde{D}_{c b}:=\delta_{b}^{a}, \tilde{D}_{a b}:=D_{a b c} \tilde{p}^{c}$, and $\alpha, \beta, \sigma$ and $\rho$ are real, $\tilde{Q}$-dependent quantities. From the very definition of $\tilde{\Delta}_{a}$ and Eq. (4.2), one obtains

$$
\begin{aligned}
\tilde{\Delta}_{a}= & \left(\tilde{p}^{0}\right)^{2}\left(\alpha^{2}+\beta^{2}\right) D_{a b c} \tilde{D}^{b e} \tilde{q}_{e} \tilde{D}^{c f} \tilde{q}_{f}+\left(\sigma^{2}+\rho^{2}\right) \tilde{D}_{a}+2 \tilde{p}^{0}(\alpha \sigma+\beta \rho) \tilde{q}_{a} \\
& +2 \tilde{p}^{0}(\alpha \rho+\beta \sigma) \tilde{q}_{b} I^{b}{ }_{a}+2\left(\tilde{p}^{0}\right)^{2} \alpha \beta D_{a b c} I^{b}{ }_{d} \tilde{D}^{d e} \tilde{q}_{e} \tilde{D}^{c f} \tilde{q}_{f} \\
& +2 \sigma \rho D_{a b c} I^{b}{ }_{d} \tilde{p}^{d} \tilde{p}^{c} .
\end{aligned}
$$

The most general BPS dyonic solution will be given by the most general solution of Eq. (4.2) with (4.6) plugged in. 
By recalling the results (3.2)-(3.5)), one can check (cfr. also the Appendix) that setting

$$
\begin{gathered}
\alpha=-\beta=-\frac{\mathbb{B}}{2 \sqrt{3} \mathbb{A}} \frac{\sqrt{\left(6 \tilde{D}-\tilde{p}^{0} A\right)^{2}-\left(\tilde{p}^{0} B\right)^{2}}}{\left(6 \tilde{D}-\tilde{p}^{0} A\right)}, \\
\sigma=\frac{\sqrt{3}\left(6 \tilde{D}-\tilde{p}^{0} A\right)}{\sqrt{\left(6 \tilde{D}-\tilde{p}^{0} A\right)^{2}-\left(\tilde{p}^{0} B\right)^{2}}}, \\
\rho=-\frac{\sqrt{3} \tilde{p}^{0} B}{\sqrt{\left(6 \tilde{D}-\tilde{p}^{0} A\right)^{2}-\left(\tilde{p}^{0} B\right)^{2}}},
\end{gathered}
$$

where

$$
\begin{aligned}
& A:=\tilde{q}_{a} \tilde{p}^{a}, \quad B:=\tilde{q}_{a} I^{a}{ }_{b} \tilde{p}^{b}, \\
& \mathbb{A}:=18 \tilde{q}_{0}+\tilde{D}^{a b} \tilde{q}_{a} \tilde{q}_{b}, \quad \mathbb{B}:=\tilde{q}_{a} I^{a}{ }_{b} \tilde{D}^{b c} \tilde{q}_{c},
\end{aligned}
$$

the supersymmetric $D 0-D 2-D 4-D 6$ black hole charge vector $\tilde{Q}$ becomes F-dual to the supersymmetric $D 0-D 4-D 6$ black hole charge vector $Q$ :

$$
\left.\tilde{Q}\right|_{(3.2]-(3.5),(4.7)-(4.11)}=\hat{Q},
$$

and all tilded quantities correspondingly become hatted. This duality trivially holds for the 'standard' BPS solution with $I_{b}^{a}=\delta^{a}{ }_{b}$, providing the BPS solution found by Shmakova 22. On the other hand, for the new, 'non-standard' solution with $I^{a}{ }_{b} \neq \delta_{b}^{a}, \tilde{x}^{a}$ changes, and so $x_{1}^{a}, x_{2}^{a}$ and $S$ do, and we find distinct solutions.

When all the brane charges are non-vanishing, the charge-dependent quantities $\mathbb{A}$ and $\mathbb{B}$ defined in (4.11) satisfy the relation

$$
\mathbb{A}\left(6 \hat{D}_{a}-\hat{p}^{0} \hat{q}_{a}\right)+\hat{p}^{0} \mathbb{B} \hat{q}_{b} I^{b}{ }_{a}=0 .
$$

Exploiting Eqs. (3.2)-(3.5), one can then write the $D 4$ brane charges $p^{a}$ of supersymmetric $D 0-D 4-D 6$ black hole in terms of supersymmetric $D 0-D 2-D 4-D 6$ black hole charges as

$$
p^{a}=\frac{\alpha \hat{p}^{0}}{\sqrt{3}}\left(\hat{D}^{a b} \hat{q}_{b}-I^{a}{ }_{b} \hat{D}^{b c} \hat{q}_{c}\right)+\frac{1}{\sqrt{3}}\left(\sigma \hat{p}^{a}+\rho I^{a}{ }_{b} \hat{p}^{b}\right) .
$$

For $n=2$ (two-moduli cubic model, treated in Sec. 2.21), by plugging (4.14) into (2.16), one can express this latter in terms of hatted charges. Thus, as for the $D 0-D 4-D 6$ case, also for the $D 0-D 2-D 4-D 6$ case one can explicitly check that the 'standard' and 'non-standard' classes of BPS solutions are supported by different, mutually exclusive domains in the charge representation space.

Finally, it is worth pointing out that if one sets $\tilde{q}^{a}=0, \alpha, \beta$ and $\rho$ vanish and $\sigma=$ $\sqrt{3}$ and then, by inserting them in Eq. (4.2), we find the most general supersymmetric $D 0-D 4-D 6$ entropy and attractor solutions in tilded charges [10]. Thus, one can conclude that (4.5) is the most general BPS Ansatz in $\mathcal{N}=2, D=4$ ungauged supergravity coupled to vector multiplets, up to the constraint (4.2). This is the full-fledged generalization of [22] for completely general BPS dyonic $D 0-D 2-D 4-D 6$ supporting charge configuration. 


\section{Conclusion}

In $\mathcal{N}=2, D=4$ ungauged supergravity coupled to vector multiplets with cubic holomorphic prepotential (2.1), by using suitable involutory matrices, we have constructed the most general supersymmetric $\left(\frac{1}{2}\right.$-BPS) attractor solution of $D 0-D 2-D 4-D 6$ extremal black hole which is Freudenthal dual to the most general attractor solution of supersymmetric $D 0-D 4-D 6$ black hole. This holds for suitable choices of the parameters $\alpha, \beta$, $\sigma$ and $\rho$ of the solution.

In an $n=2$ model with non-homogeneous moduli space, the charge representation space supporting supersymmetric $D 0-D 2-D 4-D 6$ extremal black hole attractors contains mutually exclusive domains, and inside each of these domains there exists a unique supersymmetric attractor.

Especially in the cases with more than two moduli, it would be interesting to further explore the geometric constraints on the existence of non-trivial involutory matrices satisfying (2.4)-(2.5), and thus of 'non-standard' classes of BPS attractors (which, for $n=2$, are given by the consistency condition (2.11)). The known orbit stratification of the charge representation space in models with symmetric moduli spaces would prevent the existence of 'non-standard' classes of BPS attractors; it remains to be seen whether this is also the case for homogeneous non-symmetric moduli spaces, as well. We leave this very interesting issue for further future investigation.

For what concerns the study of involutory matrices in non-homogeneous special Kähler moduli spaces arising in (the large volume limit of) Type IIA compactifications on CalabiYau manifolds, one might consider the relatively simple class of reducible cubic prepotentials, which was recently classified in [30], showing that the automorphism group in the two non-homogeneous classes of such spaces has a co-homogeneity one action, thus of a peculiar non-transitive type. We hope to report on such topics in future works. 


\section{A Appendix}

We insert $D 0-D 2-D 4-D 6$ F-dual charges $\hat{Q}$ in terms of $D 0-D 4-D 6$ charges $Q$ in the expressions made of $\hat{Q}$, using Eqs. (3.2), (3.3), (3.4), (3.5), (4.5) and (4.6). After some algebra, we find

$$
\begin{gathered}
\alpha(\hat{Q})=-\beta(\hat{Q})=-\frac{\sqrt{4 \chi^{2}-\left(2 D-q_{0}\left(p^{0}\right)^{2}\right)^{2}}}{2 \sqrt{3}\left(2 D-q_{0}\left(p^{0}\right)^{2}\right)}, \\
\sigma(\hat{Q})=\frac{2 \sqrt{3} \chi}{\sqrt{4 \chi^{2}-\left(2 D-q_{0}\left(p^{0}\right)^{2}\right)^{2}}}, \\
\rho(\hat{Q})=\frac{\sqrt{3}\left(2 D-q_{0}\left(p^{0}\right)^{2}\right)}{\sqrt{4 \chi^{2}-\left(2 D-q_{0}\left(p^{0}\right)^{2}\right)^{2}}} \\
\tilde{x}^{a}(\hat{Q})=\sqrt{3} I^{a}{ }_{b} p^{b} \\
\tilde{\Delta}_{a} \tilde{x}^{a}(\hat{Q})=3 \sqrt{3} \chi .
\end{gathered}
$$

As it is well known, the entropy is F-invariant [14, 15] (cfr. (4.11)) :

$$
\frac{S(\hat{Q})}{\pi}=\frac{S(Q)}{3 \pi\left(q_{0} p^{0^{2}}-2 D\right)} \sqrt{\frac{4}{3}(3 \sqrt{3} \chi)^{2}-9\left(4 \chi^{2}-\left(2 D-q_{0} p^{0^{2}}\right)^{2}\right)}=\frac{S(Q)}{\pi}
$$

and so are the horizon, attractor values of the moduli [15] (cfr. (4.3)-(4.4))

$$
\begin{aligned}
x_{1}^{a}(\hat{Q}) & =\frac{3}{2} \frac{I_{b}^{a} p^{b}}{3 \chi}\left(\frac{S}{\pi}\right)^{2} \frac{p^{0}}{\left(2 D-q_{0}\left(p^{0}\right)^{2}\right)}-\frac{2 \chi I_{b}^{a} p^{b}-\left(2 D-q_{0}\left(p^{0}\right)^{2}\right) p^{a}}{p^{0}\left(2 D-q_{0}\left(p^{0}\right)^{2}\right)}, \\
& =\frac{1}{p^{0}}\left(p^{a}-\frac{2 D-q_{0}\left(p^{0}\right)^{2}}{2 \chi} I^{a}{ }_{b} p^{b}\right)=x_{1}^{a}(Q), \\
x_{2}^{a}(\hat{Q}) & =-\frac{3}{2} \frac{I_{b}{ }_{b} p^{b}}{3 \chi} \frac{S(Q)}{\pi}=-\frac{1}{p^{0}} \sqrt{1-\left(\frac{2 D-q_{0}\left(p^{0}\right)^{2}}{2 \chi}\right)^{2}} I_{b}^{a} p^{b}=x_{2}^{a}(Q) .
\end{aligned}
$$




\section{References}

[1] S. W. Hawking, Gravitational Radiation from Colliding Black Holes, Phys. Rev. Lett. 26 (1971) 1344. J. D. Bekenstein, Black Holes and Entropy, Phys. Rev. D7 (1973) 333.

[2] S. Ferrara, R. Kallosh, A. Strominger, $\mathcal{N}=2$ Extremal Black Holes, Phys. Rev. D52 (1995) 5412, hep-th/9508072. A. Strominger, Macroscopic Entropy of $\mathcal{N}=$ 2 Extremal Black Holes, Phys. Lett. B383, 39 (1996), hep-th/9602111. S. Ferrara, R. Kallosh, Supersymmetry and attractors, Phys. Rev. D54 (1996) 1514, hep-th/9602136. S. Ferrara, R. Kallosh, Universality of Supersymmetric Attractors, Phys. Rev. D54 (1996) 1525, hep-th/9603090.

[3] S. Ferrara, G. W. Gibbons, R. Kallosh, Black holes and critical points in moduli space, Nucl. Phys. B500, 75 (1997), hep-th/9702103.

[4] G. W. Moore, Arithmetic and attractors, hep-th/9807087. G. W. Moore, Attractors and arithmetic, hep-th/9807056.

[5] R. Kallosh, A. D. Linde, M. Shmakova, Supersymmetric multiple basin attractors, JHEP 9911, 010 (1999), hep-th/9910021.

[6] R. Kallosh, Multivalued entropy of supersymmetric black holes, JHEP 0001, 001 (2000), hep-th/9912053.

[7] M. Wijnholt, S. Zhukov, On the uniqueness of black hole attractors, hep-th/9912002.

[8] A. Ceresole, S. Ferrara, A. Marrani, 4d/5d Correspondence for the Black Hole Potential and its Critical Points, Class. Quant. Grav. 24, 5651 (2007), arXiv:0707.0964 [hep-th].

[9] P. Dominic, T. Mandal, P. K. Tripathy, Multiple Single-Centered Attractors, JHEP 1412, 158 (2014), arXiv:1406.7147 [hep-th].

[10] T. Mandal, P. K. Tripathy, On the Uniqueness of Supersymmetric Attractors, Phys. Lett. B749, 221 (2015), arXiv:1506.06276 [hep-th].

[11] P. K. Tripathy, New Branches of Non-supersymmetric Attractors in $\mathcal{N}=2$ Supergravity, arXiv:1701.00368 [hep-th].

[12] E. Cremmer and B. Julia, The SO(8) Supergravity, Nucl. Phys. B159, 141 (1979).

[13] C. M. Hull and P. K. Townsend, Unity of superstring dualities, Nucl. Phys. B438, 109 (1995), hep-th/9410167.

[14] L. Borsten, D. Dahanayake, M. J. Duff, W. Rubens, Black holes admitting a Freudenthal dual, Phys. Rev. D80, 026003 (2009), arXiv:0903.5517 [hep-th]. 
[15] S. Ferrara, A. Marrani, A. Yeranyan, Freudenthal duality and generalized special geometry, Phys. Lett. B701, 640 (2011), arXiv:1102.4857 [hep-th].

[16] S. Ferrara, A. Marrani, E. Orazi, M. Trigiante, Dualities Near the Horizon, JHEP 1311, 056 (2013), arXiv:1305.2057 [hep-th].

[17] A. Marrani, Freudenthal Duality in Gravity: from Groups of Type $E_{7}$ to PreHomogeneous Spaces, p Adic Ultra.Anal.Appl. 7, 322 (2015), arXiv:1509.01031 [hep-th].

[18] A. Marrani, C. X. Qiu, S. Y. D. Shih, A. Tagliaferro, B. Zumino, Freudenthal gauge theory, JHEP 1303, 132 (2013), arXiv:1208.0013 [hep-th].

[19] J. J. Fernández-Melgarejo, E. Torrente-Lujan, $\mathcal{N}=2$ sugra BPS multi-center solutions, quadratic prepotentials and Freudenthal transformations, JHEP 1405, 081 (2014), arXiv:1310.4182 [hep-th].

[20] L. Borsten, M. J. Duff, S. Ferrara, A. Marrani, Freudenthal dual Lagrangians, Class. Quant. Grav. 30, 235003 (2013), arXiv:1212.3254 [hep-th].

[21] D. Klemm, A. Marrani, N. Petri, M. Rabbiosi, Nonlinear symmetries of black hole entropy in gauged supergravity, arXiv:1701.08536 [hep-th].

[22] M. Shmakova, Calabi-Yau black holes, Phys. Rev. D56, 540 (1997), hep-th/9612076.

[23] B. de Wit, A. Van Proeyen, Special geometry, cubic polynomials and homogeneous quaternionic spaces, Commun. Math. Phys. 149, 307 (1992), hep-th/9112027. B. de Wit, A. Van Proeyen, Isometries of special manifolds, hep-th/9505097.

[24] B. de Wit, F. Vanderseypen, A. Van Proeyen, Symmetry structure of special geometries, Nucl. Phys. B400, 463 (1993), hep-th/9210068.

[25] S. Hosono, B. H. Lian, S.-T. Yau, GKZ generalized hypergeometric systems in mirror symmetry of Calabi-Yau hypersurfaces, Commun. Math. Phys. 182, 535 (1996), alg-geom/9511001.

[26] R. D'Auria, S. Ferrara, String quantum symmetries from Picard-Fuchs equations and their monodromy, Annals Phys. 231, 84 (1994).

[27] A. Ceresole, S. Ferrara, A. Gnecchi, A. Marrani, d-Geometries Revisited, JHEP 1302, 059 (2013), arXiv:1210.5983 [hep-th].

[28] D. Klemm, A. Marrani, N. Petri, C. Santoli, BPS black holes in a non-homogeneous deformation of the stu model of $\mathcal{N}=2, D=4$ gauged supergravity, JHEP 1509, 205 (2015), arXiv:1507.05553 [hep-th].

[29] S. Ferrara, E. G. Gimon, R. Kallosh, Magic supergravities, $\mathcal{N}=8$ and black hole composites, Phys. Rev. D74, 125018 (2006), hep-th/0606211. 
[30] V. Cortés, M. Dyckmanns, M. Jüngling, D. Lindemann, A class of cubic hypersurfaces and quaternionic Kähler manifolds of co-homogeneity one, arXiv:1701.07882 [math.DG]. 